\title{
Affective Computational Priming and Creativity
}

\author{
Sheena Lewis ${ }^{1}$, Mira Dontcheva ${ }^{2}$, Elizabeth Gerber ${ }^{1,3}$ \\ Technology and Social Behavior ${ }^{1}$, Segal Institute of Design ${ }^{3}$ \\ Northwestern University \\ Evanston, IL 60208 USA \\ sheena@u.northwestern.edu, egerber@northwestern.edu \\ Advanced Technology Labs ${ }^{2}$ \\ Adobe Systems \\ San Francisco, CA 94103 USA \\ mirad@adobe.com
}

\begin{abstract}
While studies have shown that affect influences creativity, few investigate how affect influences creative performance with creativity support tools. Drawing from methods commonly used in psychology research, we present affective computational priming, a new method for manipulating affect using digitally embedded stimuli. We present two studies that explore computational techniques for inducing positive, neutral, and negative affect and examine their impact on idea generation with creativity support tools. Our results suggest that positive affective computational priming positively influences the quality of ideas generated. We discuss opportunities for future HCI research and offer practical applications of affective computational priming.
\end{abstract}

\section{Author Keywords}

creativity support tools, design, computational primes

\section{ACM Classification Keywords}

H5.m. Information interfaces and presentation (e.g., HCI): Miscellaneous.

\section{INTRODUCTION}

Creative professionals, such as engineers, scientists, and designers, increasingly rely on technology to complete creative tasks at work. They use web browsers to conduct research and search for inspiration. They document projects on wikis in order to collaborate with others. They depend on graphic design software and word processors to design and create. In response, human-computer interaction (HCI) researchers have started building creativity support tools, or software to enhance creative performance [37].

To inform the design of creativity support tools, HCI researchers have turned to psychological research on the cognitive aspects of individual and group creativity. For example, Hewett suggests that creativity support tools should allow users to formulate, re-formulate, and solve problems as their domain or state of the problem changes [20]. While this perspective has spawned new software and interfaces that enhance the cognitive variation necessary for

Permission to make digital or hard copies of all or part of this work for personal or classroom use is granted without fee provided that copies are not made or distributed for profit or commercial advantage and that copies bear this notice and the full citation on the first page. To copy otherwise, or republish, to post on servers or to redistribute to lists, requires prior specific permission and/or a fee.

CHI 2011, May 7-12, 2011, Vancouver, BC, Canada.

Copyright 2011 ACM 978-1-4503-0267-8/11/05...\$10.00.
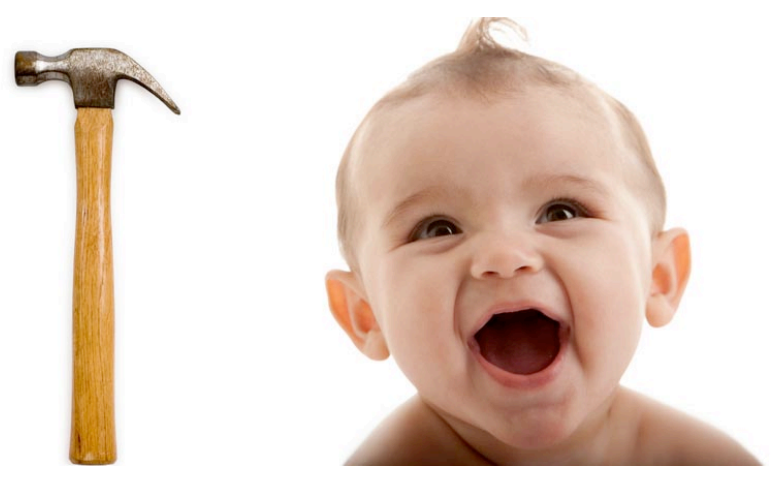

Figure 1. Our studies show that a picture of a hammer (left) induces neutral affect, while a picture of a laughing baby (right) induces positive affect. Positive affect positively influences the quality of ideas generated in a creativity task.

creativity [37], few HCI researchers have yet to take an affective perspective on creativity, despite extensive psychological research in the domain [e.g. 14, 23].

Affect, the experience of emotion [42], has been shown to be a source of the cognitive variation necessary for creativity $[4,15,22]$. In the laboratory, experimenters induced positive affect by showing participants funny videos or giving them candy bars and asked them to perform established creativity tasks, such as generating alternative uses for a brick [18]. These studies show that positive affect leads to increased creative performance. But does positive affect lead to increased creative performance with real-world creativity support tools? How might we induce positive affect in a more contextualized and unobtrusive way?

In this paper we present two studies that demonstrate that affect has a positive influence on creativity support tool use. We introduce affective computational priming, a new method for manipulating affect using digitally embedded stimuli that influence a later response. In our studies, the embedded stimuli are images (see Figure 1), and we study their influence on creative idea generation tasks. In a first study with 240 participants generating unusual uses for common objects [18], we find that affective computational priming influences creative performance and that embedded images can be used as affective computational primes. Images are already part of creativity support tools in the form of sample materials, tutorials, and startup screens, and 
therefore, they offer an opportunity for studying the link between affect and creativity in a contextualized unobtrusive way. In a second study with 31 participants and an existing creativity support tool, a sketching application, we find that positive affective computational priming positively influences the quality of created sketches. This study is the first to empirically demonstrate how computationally induced affect influences creative performance in the context of a creativity support tool.

This research contributes to design and HCI by introducing psychological research on affect to creativity support tool research, demonstrating the effectiveness of affective computational priming, and providing design implications for creativity support tools.

\section{BACKGROUND}

Creativity is defined as the generation of novel and useful ideas [2]. According to Simonton's [38] evolutionary theory of creativity, the probability of generating novel ideas increases with the number and breadth of cognitive elements available for association and considered relevant to the problem. For example, when developing an interface for CAD software, the likelihood of generating a novel interface increases if the designer combines a broad number of insights gathered from architects, engineers, construction managers, builders, etc. rather than a few insights generated from a single user. The more insights used to inform the new design, the greater likelihood that a novel idea will emerge [38]. Because creativity depends on novelty, which is a function of cognitive variation, increasing variation is likely to increase creativity. Psychologists find that affect supports cognitive variation leading to creativity $[4,15,22]$. For example, Isen and colleagues found that when subjects were primed for positive affect, they exhibited a more original and diverse range of word associations as compared to control subjects [24].

While HCI researchers have designed creativity support tools to increase cognitive variation in order to improve creative performance, few have considered the use of affective stimuli in creativity support tools.

\section{Affect and Creativity}

Affect is the experience of emotion that can occur without extensive perceptual and cognitive encoding and is often the first reaction to a given stimuli [42]. Affect constitutes the primary motivational system for human beings, modifying, controlling, and directing cognitive processes and task performance [26]. Affect may have two forms: trait (stable) or state (temporary); the latter may be induced by external stimuli [26].

Isen [22] proposes that positive affect influences cognitive activity in three ways. First, positive affect increases the number of cognitive elements available for processing, thereby increasing the number of elements available for association. Second, positive affect supports defocused attention, therefore broadening attention and leading to an increase in the numbers of cognitive elements treated as relevant to the problem. Third, positive affect increases cognitive flexibility, thereby increasing the probability of association between diverse cognitive elements [22, 23].

Similarly, Fredrickson [15] proposes a broaden-and-build model of positive emotion, which proposes that positive emotions (such as joy, contentment, interest, and love) broaden a person's scope of attention and cognition, increasing the number of available cognitive elements and breadth of those elements relevant to the problem. According to Simonton's evolutionary theory [38], breadth of relevant cognitive elements should increase creative performance.

In laboratory studies, Isen and colleagues have established an extensive repertoire (see [22] for a review) of ways to experimentally induce state (temporary) positive affect. In Isen's studies, participants are most often given gifts and treats, shown a clip of a comedy film, or played an excerpt from affect-laden music. For example, in two separate experiments with college students, participants were shown a clip of the movie Gag Reel or given a small bag of candy to induce affect [23]. In both cases, positive affect improved performance on creative ingenuity tasks, such as Mednick's Remote Associates Task ${ }^{1}$ [31] and Duncker's Candle Task $^{2}$ [12]. This study suggests the possibility of HCI researchers designing creativity support tools to induce positive affect as a way of supporting idea production.

Positive affect has also been shown to influence performance by influencing the cognitive processes underlying motivation [9]. Scholars posit that positive affect influences expectancy (belief that effort will result in good performance), which in turn influences goal commitment, goal setting, persistence, and performance [13]. In a study of 97 undergraduate students, researchers induced positive affect by giving the students a bag of candy and then asked them to solve 10 anagrams. The study found that participants induced with positive affect performed better, were more persistent, tried harder, and reported higher levels of motivation than those in the neutral condition who did not receive a bag of candy.

Based on this literature, we formulated the following hypotheses:

\section{Hypothesis 1}

People who are computationally primed for positive affect will generate a greater number of ideas and ideas that are rated as more creative than those primed for negative or neutral affect.

\footnotetext{
${ }^{1}$ In Mendick's Remote Associates Task, participants are given three words and asked to give a fourth word that relates to the original three words.

2 In Duncker's Candle Task, participants are given a candle, a book of matches, and a box of thumbtacks and asked to fix a lighted candle to a corkboard such that the candle wax won't drip on the table.
} 


\section{Hypothesis 2}

People who are computationally primed for positive affect will generate a greater number of ideas and ideas that are rated as more creative than those who are not computationally primed.

Next, we discuss our study design and then describe our experiments in detail.

\section{STUDY DESIGN}

To study the influence of affect on creative performance with creativity support tools, we chose to use images as affective computational primes, because images are commonly used in startup screens, sample materials, and learning resources in modern software applications. We first created a dataset of affect-laden images and evaluated their influence on a common creativity task used in psychology, Guilford's Alternative Uses Task [18], to confirm that affective computational primes influence creativity. In Guilford's Alternative Uses Task, participants are asked to generate as many unusual uses as possible for a common object (e.g., brick).

In a second experiment, we embedded the same affectladen images in a creativity support tool, the digital sketching application Adobe Ideas, and studied the impact of positive, neutral, and negative computational primes on idea generation in another common creativity task, the Torrance Test of Creativity Circles [41]. In this task participants are asked to sketch as many real objects as possible incorporating a circle.

We chose Guilford's Alternative Uses Task [18] and the Torrance Test of Creativity Circles [41], because they have been extensively developed and validated as a measure of ability to generate ideas in hundreds of studies on creativity over the past 30 years [30].

\section{Amazon Mechanical Turk}

To create a dataset of affect-laden images and to carry out our first experiment, we made use of Amazon Mechanical Turk. Mechanical Turk is an micro-task market where people, referred to as Turkers, use the web to complete one or more small tasks in exchange for micro-payments that typically range from $\$ 0.01$ to $\$ 0.10$ per task. Mechanical Turk has been shown to be an effective platform for crowdsourcing users studies [28]. Hence, we use Mechanical Turk in our studies, because it provides low recruitment costs and immediate access to hundreds of participants. While limitations of Mechanical Turk do not allow us to verify accurate demographic information of the participants, previous studies [21, 35, 39] have reported that the demographics of Mechanical Turkers are similar to typical Internet users, i.e., the majority are between 18-40 years old and slightly more educated than the general US population. The ratio of male to female is $45 \%$ to $55 \%$.

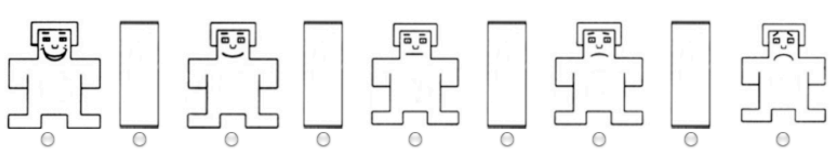

Figure 2. Self-Assessment Manikin [29] used to rate emotion

\begin{tabular}{|c|c|c|c|}
\hline Affect & $\begin{array}{c}\text { Image } \\
\text { Description }\end{array}$ & $\begin{array}{l}\text { IAPS Rating } \\
\text { Mean (Std. Dev.) }\end{array}$ & $\begin{array}{c}\text { MTurk } \\
\text { Rating } \\
\text { Mean (Std. Dev.) }\end{array}$ \\
\hline \multirow[t]{12}{*}{ Positive } & Laughing baby & $8.20(1.31)$ & $8.65(0.67)$ \\
\hline & Puppy & $8.34(1.12)$ & $8.25(1.16)$ \\
\hline & Dessert & $7.53(1.73)$ & $8.15(1.31)$ \\
\hline & Beach & $8.22(1.08)$ & $8.00(1.62)$ \\
\hline & Bunny & $7.89(1.26)$ & $7.90(1.33)$ \\
\hline & Bride & $7.64(1.46)$ & $7.85(1.46)$ \\
\hline & Couple & $7.80(1.55)$ & $7.70(1.38)$ \\
\hline & Mountain lake & $7.27(1.59)$ & $7.45(1.50)$ \\
\hline & Sunflower & $7.16(1.56)$ & $7.45(1.23)$ \\
\hline & Salsa & $7.25(1.83)$ & $7.20(1.47)$ \\
\hline & Mountain top & $7.61(1.46)$ & $6.90(1.55)$ \\
\hline & Athlete & $7.09(1.36)$ & $6.45(1.57)$ \\
\hline \multirow[t]{10}{*}{ Neutral } & Cutlery & $5.27(1.09)$ & $6.85(1.46)$ \\
\hline & Shoes & $4.82(1.20)$ & $6.80(1.70)$ \\
\hline & Umbrella & $4.72(1.00)$ & $6.65(1.57)$ \\
\hline & Clothes rack & $4.82(0.99)$ & $6.10(1.52)$ \\
\hline & Stool & $4.63(1.17)$ & $6.00(1.38)$ \\
\hline & File cabinet & $4.45(1.36)$ & $5.90(1.97)$ \\
\hline & Hammer & $4.95(0.87)$ & $5.70(1.34)$ \\
\hline & Factory worker & $4.87(1.06)$ & $5.55(1.70)$ \\
\hline & Secretary & $4.72(1.36)$ & $5.50(1.43)$ \\
\hline & Trashcan & $4.43(1.16)$ & $5.45(1.73)$ \\
\hline \multirow[t]{11}{*}{ Negative } & Oil spill & $3.89(1.58)$ & $3.60(2.16)$ \\
\hline & Natural disaster & $3.49(1.87)$ & $3.30(2.15)$ \\
\hline & Medical & $3.28(1.64)$ & $2.80(1.79)$ \\
\hline & Homeless & $2.87(1.28)$ & $2.58(1.26)$ \\
\hline & Dead body & $2.78(1.71)$ & $2.50(1.79)$ \\
\hline & Explosion & $1.62(1.20)$ & $2.45(1.43)$ \\
\hline & Stitches & $2.06(1.23)$ & $2.25(1.48)$ \\
\hline & Deformed & $2.26(1.57)$ & $2.15(1.53)$ \\
\hline & Burn victim & $1.79(1.30)$ & $2.05(2.19)$ \\
\hline & Dead pet & $1.97(1.16)$ & $1.52(1.10)$ \\
\hline & Dead bodies & $1.53(1.25)$ & $1.50(1.31)$ \\
\hline
\end{tabular}

Table 1. We created an image database for our experiments using Mechanical Turk. The Mechanical Turk ratings were consistent with the IAPS database ratings.

\section{Stimuli}

To select images that induce positive, neutral, and negative affect, we referenced the International Affective Picture System (IAPS) manual. IAPS is a database of images for experimental investigations of affect [29]. We created our own database of affect-inducing images, because IAPS administrators stipulate that images from the IAPS database cannot be posted on the Internet or shared with anyone outside of the study participants. To create our own dataset, we selected images from iStockphoto.com based on the image descriptions (e.g., "laughing baby") in the IAPS manual and gathered ratings for each image using the 9point Self-Assessment Manikin (SAM) Scale [29]. Figure 2 shows SAM, which uses graphical figures to depict human 
emotions ranging from smiling (i.e. happy) to frowning (i.e. unhappy). The blocks between each figure allow participants to indicate if their emotion falls between two figures. After viewing the instructions on how to use SAM, participants were shown an image for five seconds and then asked to "Select the figure that most closely corresponds to how you felt viewing the previous image" using SAM. Our rating method is similar to the method used to generate the IAPS affect image ratings.

The main difference between our rating approach and the approach employed by Lang et al. [29] is that we collected ratings using Mechanical Turk, while they collected ratings in a laboratory setting. We collected data for 33 images (12 positive, 10 neutral, 11 negative), and each image received an average of 20 ratings. To validate that the ratings were genuine, we asked participants to complete a validation test. After users rated an image, they were asked to describe the image they were rating and the reasons for their rating. Participants who failed the validation test were excluded from our analysis. Table 1 compares our image ratings to the IAPS database.

\section{EXPERIMENT 1: ALTERNATIVE USES TASK}

While our broader goal is to understand the influence of affect on the use of creativity support tools, in our first experiment, we wanted to determine whether affective computational primes have a similar impact as previously studied primes. Thus, for our first experiment, we followed previous experiments and studied an established creativity task, Guilford's Alternative Uses Task. We asked participants to generate unusual uses for two common objects, a brick and a quarter. The brick is frequently used as the object in the unusual uses task, but because we were concerned that participants may use one of the numerous websites that list unusual uses for a brick, we chose to also use a quarter as the object, which to our knowledge has not been used in such experiments.

\section{Participants}

We recruited 240 participants from Mechanical Turk that reside in the United States and had successfully completed at least $80 \%$ of prior Amazon Mechanical Turk tasks. Participants were either paid $\$ 0.05$ for completing the brick task or $\$ 0.50$ for the quarter task. Consistent with previous findings [19], we found that offering people more money did not influence the quality of the data but did influence the rate at which Turkers responded, thereby allowing us to collect data more quickly for the quarter task.

\section{Measures}

Researchers have long debated how to best assess creativity in a way that consistently captures the complex dimensions of creativity [34]. The most commonly adopted approach $[1,5,17]$ and the one we employ is to ask domain experts to subjectively make scale-rated assessments of creativity. Typically, domain experts rate originality, feasibility, elaboration, and flexibility and count the number of ideas generated [3].

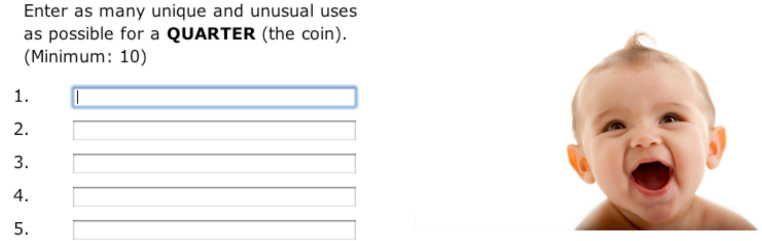

Figure 3. Screenshot of the unusual uses task on Mechanical Turk for the positive condition.

\section{Procedure}

Each Turker was assigned to one of four conditions: positive affect, neutral affect, negative affect, and no affect (our baseline). The instructions on completing the task (adopted from [18]) were as follows:

In this task, your goal is to think of as many unique and unusual uses for a common object. For example, using a paper clip as an earring is an unusual and unique use. However, using a paper clip to bind papers is not unique or unusual. Try to think of as many unique and unusual uses as possible. DO NOT use any external sources (e.g., websites, people) to complete this task.

To the right of the instructions was a 350 x 233 pixel image. This image served as the affective computational prime and varied under the different conditions. For the baseline, we did not include an image. We did not provide information about why the image was present. Using our database of affective images, we selected the image with the highest rating (a laughing baby) and the lowest rating (dead bodies after the 2010 earthquake in Haiti) for the positive and negative conditions, respectively. For the neutral condition, we selected the image with a rating closest to the median rating of 5 and with the lowest standard deviation (a hammer).

To proceed, Turkers clicked a next button. They were then presented with a web form (see Figure 3), which asked them to enter as many unique and unusual uses for an object (either a brick or a quarter) as possible. They were required to enter a minimum of 10 responses but had the option of entering up to 20 responses. The affective computational prime remained visible throughout the task.

Each Turker either completed the task for a brick or a quarter, not both. For the brick task, we collected responses from 20 Turkers for each condition (positive, negative, neutral, and baseline). For the quarter task, we collected responses from 40 Turkers for each condition.

\section{Analysis}

Out of the 240 total Turkers recruited on Mechanical Turk, we excluded responses from 14 Turkers, because they did not follow directions. The remaining 226 Turkers (75 in the brick task; 151 in the quarter task) produced 2,463 responses (836 for the brick task and 1627 for the quarter task). Though previous studies [19] suggest task completion times provided by Mechanical Turk are unreliable, we note that the average completion time was six minutes. 


\section{Creativity}

We collected expert ratings for all responses. For the brick task, we collected ratings from two expert raters. Both were members of the research team and each has over 10 years experience as design professionals.

For the quarter task, we collected ratings from three expert raters, who we recruited through an e-mail distribution list of a graduate design program on the west coast in the United States. Two were current design graduate students, who have worked as design professionals for at least 4 years, and the other was an alumni, who had 8 years of experience as a design professional.

All expert raters were blind to the conditions. Inter-rater reliability for the raters of the brick and quarter tasks were Cohen's Kappa $=0.77,0.68(\mathrm{p}<0.01,0.05)$ respectively.

We asked the expert raters to rate each response on a scale of 1-5 according to originality, feasibility, elaboration, and flexibility [18]. Originality refers to how unique the response is as compared to others. Feasibility refers to whether the described use is possible. Elaboration refers to the amount of detail in a response. Flexibility refers to the variety of categories for all of the responses given by a particular participant. For example, the raters rated using a brick as "a doorstop" with 1 for originality because most participants also generated this use for a brick, 5 for feasibility because the use of a brick as a doorstop is feasible, 1 for elaboration because there is minimal detail included in the description of the use, and 5 for flexibility because the participant suggested no other related ideas within their own set of responses. In contrast, the raters rated using a brick "to mark a spot where a treasure is buried" with 4 for originality, 5 for feasibility, 4 for elaboration, and 5 for flexibility because there were no other ideas within the participant's response that were related to using a brick as a marker.

In order to calibrate their ratings, the raters first rated a small subset of the responses and discussed their ratings until they agreed on a strategy for rating. After they reached agreement, the expert raters continued rating their portion of the responses. The brick expert raters each rated approximately 450 responses. The quarter expert raters each rated approximately 600 responses.

\section{Results}

Our data suggests that although the effect is not very large, positive affect-laden images positively influence idea generation in both the brick and quarter tasks, validating the influence of affective computational primes on creative performance. We used Welch's t-test to measure significance for all reported findings and report mean ratings $(\mu)$ and their standard deviations $(\sigma)$.

\section{Quality of Ideas}

As predicted in Hypothesis 1 and 2, positive affect positively influenced the quality of ideas. For both the brick and quarter tasks, we find that Turkers who were computationally primed to feel positive affect generate ideas rated as more original than those primed for neutral affect. The responses from Turkers primed with positiveladen stimuli were rated as significantly more original (brick: $\mu=2.76 \sigma=1.31$, quarter: $\mu=2.76 \sigma=0.96$ ) than the responses from participants primed to feel no affect in the neutral condition (brick: $\mu=2.58 \sigma=1.22, p<0.04$, quarter: $\mu=2.56 \sigma=0.94, p<0.008)$. Surprisingly, the responses from Turkers primed with negative-laden stimuli were also rated as significantly more original (brick: $\mu=2.85 \sigma=1.30$, quarter: $\mu=2.71 \sigma=0.87)$ than those primed to feel neutral affect (brick: $\mu=2.58 \sigma=1.22, p<0.05$, quarter: $\mu=2.56$ $\sigma=0.94, \mathrm{p}<0.02$ ).

In the brick task, we find that Turkers who are computationally primed with positive affect generate ideas rated as significantly more original $(\mu=2.76 \sigma=1.30)$ than those who are not computationally primed $(\mu=2.54 \sigma=1.32$, $\mathrm{p}<0.02$ ). Additionally, Turkers who were computationally primed for negative affect in the brick task also generate ideas that were rated as significantly more original $(\mu=2.85$ $\sigma=1.30)$ than those who are not computationally primed $(\mu=2.54 \sigma=1.32 \mathrm{p}<0.03)$.

In the quarter task, we did not find significant differences between the positive and baseline conditions. Additional data is needed to understand this difference between the brick and quarter tasks.

In both tasks, we did not find significant differences in the feasibility, elaboration, and flexibility ratings.

\section{Quantity of Ideas}

We did not find significant differences in the number of ideas generated between the positive, neutral, negative, and baseline conditions (Hypothesis 1 and 2).

\section{EXPERIMENT 2: CREATIVITY SUPPORT TOOLS}

The goal of the second experiment was to examine how computationally primed affect influences creativity with an existing creativity support tool. Since creativity support tools on the desktop can be complex, for our experimental platform, we selected a simple sketching application (Adobe Ideas) on a tablet (iPad). We felt the tablet was a particularly useful platform to explore, as creative professionals are increasingly using tablets to generate and capture ideas.

\section{Participants}

We recruited 31 participants (12 women) through email distribution lists at a small college and medium-size software company located on the west coast of the United States. Data from four participants was removed (2 did not follow instructions, 2 were familiar with the task prior to the study). The remaining 27 participants ( 9 women) ranged in age (24-56 years old) and occupation (engineers, designers, managers, IT specialists, and marketing professionals). Two participants owned or had extensive experience using a tablet PC. Four participants had used Adobe Ideas prior to the study. Participants were given \$20 gift cards for their participation. 


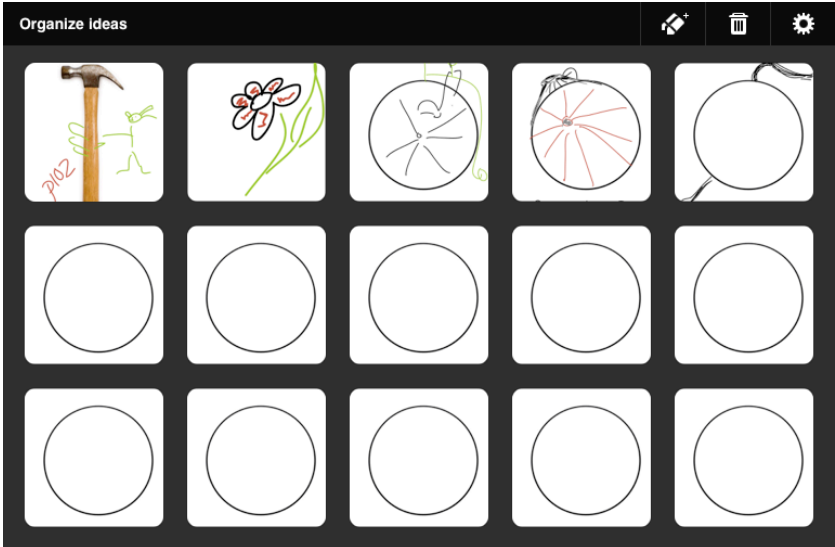

Figure 4. This screenshot shows a portion of the screen for a completed circles task in the neutral condition. The participants were given 20 circles as part of the task.

\section{Stimuli}

To induce positive, neutral, and negative affect, we used the same images as in Experiment 1.

\section{Measures}

Creativity. Expert raters assessed originality, elaboration, and flexibility and counted the number of sketches generated by each participant.

\section{Procedure}

The participants were randomly assigned to one of the four conditions: positive affect, neutral affect, negative affect, and no affect (baseline). Each study session included two to four participants.

First, the participants completed a web survey on the iPad that included measures for creative efficacy [40] and confidence levels using a tablet (adapted from [10]) as well as basic demographic information. The study moderator then led the participants through a brief tutorial on how to use the sketching software including information about how to draw, erase, scale, undo, and change the color/size of the pen. After participants confirmed that they felt comfortable using the tool, their mood was accessed using the SAM mood rating. Participants in the positive, neutral, and negative conditions were told about an additional feature of the software, which allows images to be used as a background for a drawing. They were instructed on how to insert an image in their drawing from the photo library. Each participant's library depended on the condition to which they were assigned. The participants in the positive condition had a library that included only positive affectinducing images, while participants in the negative condition saw only negative affect-inducing images in their photo library.

Participants were told to select the photo of the laughing baby in the positive condition, the photo of the hammer in the neutral condition, and the photo of the dead bodies in Haiti in the negative condition. Participants in the baseline condition were not told how to embed images in the background of a drawing. After drawing on the embedded
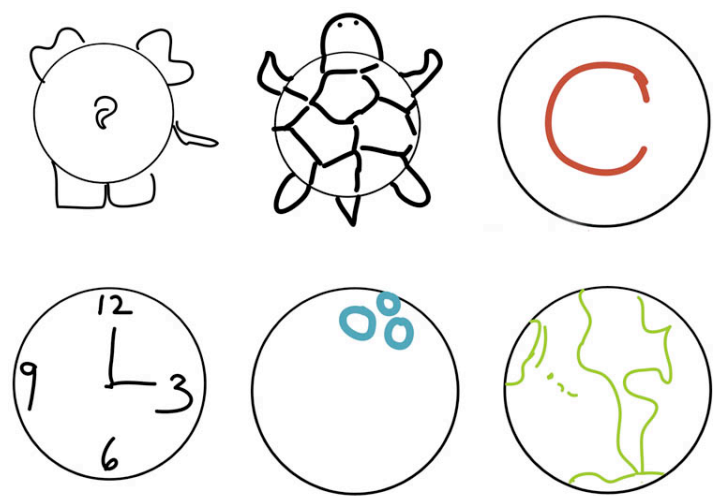

Figure 5. Sketches of an elephant, a turtle, and a copyright sign (top) received high originality ratings, while sketches of a clock, bowling ball, and globe received low originality ratings (bottom).

image for approximately one minute, the participants were told it was time to start the study. We replicated one of the Torrance Creative Thinking Tests [41], the circles task. The participants were given the following written and verbal instructions.

"For the next 5 minutes, draw as many sketches as you can using the circles provided. Avoid drawing abstract sketches. Be sure to use the circles as a part of the picture (not just a border around a picture).

1. Draw as many sketches as possible.

2. Make the sketches as unusual or different as you can.

3. Quality (or "prettiness") doesn't matter.

The participants were given 20 circles as part of the task (see Figure 4) and after five minutes they were asked to stop. In contrast to Experiment 1, the participants only saw the affect-laden images during the tutorial and not during the sketching task. After completing the task, participants were again asked to complete the SAM affect survey. They were asked if they had ideas about the reason for the study. Only two indicated that they had previous knowledge of the task (data from these participants was removed).

\section{Analysis}

We collected 226 sketches with 72 from the positive condition, 56 from the neutral, 46 from the negative, and 52 from those who were not primed.

One expert rater who was blind to the conditions rated the originality, elaboration, and flexibility of all the sketches. A second rater rated $10 \%$ of the sketches, and since the inter-rater reliability between the two raters was high (Cohen's Kappa $=0.83 \mathrm{p}<0.001$ ), we used ratings from the first rater. Both raters were members of the research team. Figure 5 shows sketches with high and low originality ratings. 


\begin{tabular}{|l|c|c|c|c|}
\hline Condition & $\begin{array}{c}\text { Total } \\
\text { Participants }\end{array}$ & $\begin{array}{c}0-2 \\
\text { Creative } \\
\text { Sketches }\end{array}$ & $\begin{array}{c}3-5 \\
\text { Creative } \\
\text { Sketches }\end{array}$ & $\begin{array}{c}6-8 \\
\text { Creative } \\
\text { Sketches }\end{array}$ \\
\hline Baseline & 7 & 4 & 3 & 0 \\
\hline Negative & 6 & 2 & 4 & 0 \\
\hline Neutral & 7 & 2 & 4 & 1 \\
\hline Positive & 7 & 0 & 3 & 4 \\
\hline
\end{tabular}

Table 2. The distribution of participants with respect to the number of creative sketches they generated.

\section{Results}

Our results indicate that positive affect positively influences the quality (originality and elaboration) of ideas generated. The results did not show any correlation to the age, gender, or occupation of the participants; nor was previous experience using tablets or Adobe Ideas related to the results.

\section{Quality of ideas - Originality}

As predicted in Hypothesis 1, people primed to feel positive affect generated sketches rated as significantly more original $(\mu=2.65 \sigma=1.2)$ than those primed to feel negative affect $(\mu=2.15 \sigma=1.13, p<0.02)$.

As predicted in Hypothesis 2, people primed to feel positive affect created sketches rated as significantly more original $(\mu=2.65 \sigma=1.2)$ than those who were not primed $(\mu=2.03$ $\sigma=1.12, \mathrm{p}<0.001)$.

We also found that those in the neutral condition generated sketches rated as significantly more original $(\mu=2.66$ $\sigma=1.18)$ than those in the negative $(\mu=2.15 \sigma=1.13)$ and baseline $(\mu=2.03 \sigma=1.12)$ conditions ( $p$-value $<0.02,0.001$, respectively).

In a deeper analysis, we examined the distribution of originality ratings for the drawings of each participant. Specifically, we calculated the number of sketches that were rated 3 or above (on a 5-point scale with 5 being extremely original) for each participant. For the rest of this discussion, we refer to sketches with an originality rating of 3 or above as creative. Table 2 shows the distribution of creative sketches per person across the four conditions.

Those primed to feel positive affect generated the most creative sketches. All participants in the positive condition generated at least 3 creative sketches. In fact, four out of seven of the participants in the positive condition created between 6 and 8 creative sketches. In contrast, in the baseline condition four out of seven participants created between 0 and 2 creative sketches. Although we did not see big differences between the number of participants who had creative sketches in the neutral and negative conditions, we suspect that a larger sample will illustrate that negative affect leads to fewer participants with creative sketches than neutral affect.
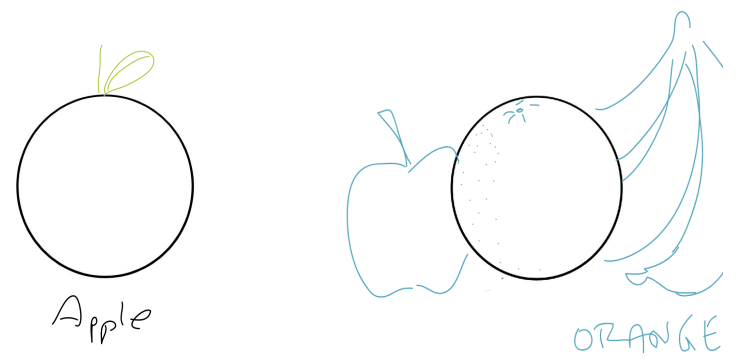

Figure 6. Example of a sketch with a low elaboration rating (left) and a sketch with a high elaboration rating (right).

Quality of Ideas - Elaboration

In addition to differences in originality, we observed differences in the amount of elaboration per condition (see Figure 6). Consistent with past research, we find that positive affect expands the number of cognitive elements associated with a task, supporting elaboration. Those primed for positive affect were more likely to elaborate $(\mu=2.67 \sigma=0.96)$ than those primed for negative affect $(\mu=2.04 \sigma=0.69)$ and those who were not primed $(\mu=2.13$ $\sigma=0.95, p$-value $<0.001,0.001$, respectively). As shown in Figure 6, elaboration includes drawing additional elements, such as bananas and apples, in addition to the primary response, the orange.

Those in the neutral condition $(\mu=2.49 \sigma=1.00)$ also elaborated on their sketches significantly more than those in the negative $(\mu=2.04 \sigma=0.69)$ and baseline $(\mu=2.13 \sigma=0.95)$ condition (p-value $<0.01,0.04$, respectively).

Quantity of Ideas

People primed to feel positive affect created more sketches $(\mu=10.29 \sigma=4.27)$ than those primed to feel negative $(\mu=7.66 \sigma=3.27)$ and neutral $(\mu=8.00 \sigma=4.12)$ affect as well as those who were not primed $(\mu=7.43 \sigma=2.76)$. Although the differences are not significant $(p<0.21$ for negative, $\mathrm{p}<0.25$ for neutral, $\mathrm{p}<0.15$ for baseline), there are trends that suggest that people in the positive condition may generate a larger quantity of sketches. While this result is consistent with research that finds that positive affect influences persistence and performance $[8,9]$, more data is needed to verify these trends.

\section{DISCUSSION}

Consistent with past research on affect and creativity [22] we found that positive affect positively influences the quality of ideas generated when using a creativity support tool. Psychology researchers reason that positive affect leads to improved creative performance by increasing the number of cognitive elements available for processing and association as well as by broadening attention to those elements treated as relevant to the problem [22] and supporting goal-setting, expectancy, and persistence during a task [13]. Somewhat surprisingly, in Experiment 2 we also found that neutral affect also has a positive influence on the quality of ideas generated, while negative affect is indistinguishable from the baseline condition. This implies 
that the effect is not due to the presence of images but is tied to the content of the image. Although the mean creativity ratings for the neutral and positive are similar, the distribution of creative sketches across participants for the two conditions is quite different; hence, we suspect that the positive-laden prime may have a stronger effect. We plan to study this further in future work.

Also, in Experiment 1 we unexpectedly found that negative affect also positively influences creative performance. While most experimental studies of affect find a positive relationship between experimentally induced positive affect and creative performance, a few studies find that both positive and negative affect support creativity. Kaufmann and Vosburg [27] suggest that positive affect supports a satisficing solution strategy and negative affect supports an optimizing solution strategy, both of which are useful for creative problem solving. We suspect that the nature of the platform may influence the effect of negative primes. People may be less sensitive to negative primes on the web, because they commonly see such images through news and entertainment channels, while negatively-laden images are uncommon in design applications.

Our experiments employed two different types of computational primes: passive and active. In Experiment 1, the computational primes were embedded as background images and user interactions with the primes were passive, as we did not force any direct user interaction with the images. On the other hand, in Experiment 2, the computational primes were part of instructional tutorials, and the participants were asked to draw on top of the images, thereby actively engaging with the primes. The active computational priming technique offers a higher guarantee that the user sees the affect-laden image; but users may not actively engage in such an instructional tutorial before every use. We suspect that active affective computational primes may have a stronger impact on creativity; however, more research is necessary to confirm this hypothesis.

Collectively, our findings demonstrate that affective computational primes, or interaction embedded stimuli, influence technology use. To our knowledge, this is the first investigation to demonstrate this effect. While web-based companies are increasingly using A-B testing to make small manipulations that improve their websites, it is not always understood how and why such design changes influence behavior. We provide a theoretical explanation and empirical demonstration of how affect could serve as a mechanism for influencing human-computer interaction.

\section{Design Implications for Creativity Support Tools}

Since our data shows that computationally primed positive affect positively influences the quality of ideas generated during creativity tasks, designers of creativity support tools should consider the affective influences of all materials associated with tools including startup screens, background images, sample material, and learning content. To test for the affective nature of materials, designers can make use of crowd-sourcing platforms such as Mechanical Turk.

Furthermore, given reported practices regarding multitasking [11], it is quite possible that users are engaged with multiple applications at once and may be influenced by them. For example, reading the news or answering email while engaging in a creative task may have an unanticipated influence on a user's creativity. Therefore, designers of creativity support tools should consider ways to reengage users after task switching during a creative task.

Since a link between affect, goal-setting, expectancy, and persistence has been established by others [9, 13] and demonstrated in our experiments, affect-laden primes may offer a technique for re-engaging users when productivity declines. Alternatively, affect may also be useful as a consideration for adaptive user interface models that not only predict user needs in order to optimize productivity, but also offer affect-appropriate suggestions. Including affect in computational models is part of research in affective computing, which aims to understand how computers can be more emotionally intelligent to reduce people's negative emotions [33].

\section{LIMITATIONS}

As other scholars have acknowledged [34], there are concerns with using expert raters to assess creativity, as their ratings can be subjective and ultimately taint the results. While we are satisfied with inter-rater reliability and reliance on extensive and established means for rating creativity, we intend to consider crowd-sourcing creativity ratings to acquire aggregate creativity ratings rather than relying on the opinions of a few raters.

Although Amazon Mechanical Turk has many benefits to researchers, because it is a low cost, low time commitment, and short turnaround platform, it may not be the best platform for creativity studies. There are two factors that had an impact on our experiments. First, because the platform is web-based, Turkers have access to other resources and can readily turn to the Web to search for answers. Guilford's Unusual Uses Task (Experiment 1) is commonly done with a brick, and there are a number of websites with solutions. This makes it difficult to evaluate whether a participant generated all of the responses or copied some or all of them from external sources. Second, creativity is linked to domain expertise, which increases over time [1], but Turkers are not incentivized to spend a long time completing a task, but rather to complete many small tasks quickly in order to make more money. It remains to be seen whether crowd-sourcing platforms are environments appropriate for creative tasks.

\section{FUTURE RESEARCH}

Our study suggests a number of areas for future research. First, we plan to explore different techniques for integrating affective computational primes into existing creativity support tools. Affective images, for example, could be placed on the start-up screen or help menu of a creativity 
support tool. Other stimuli that could be manipulated to induce affect include colors, textures, and sound. For example, a computational prime for a search tool might involve affect-laden search icons. Additionally, many software programs currently include videos in tutorials that instruct users on new features. Videos may turn out to be stronger primes because they stimulate both the visual and auditory senses.

Affect has been shown to influence user behavior in other domains beyond creativity. Affect has been empirically related to the evaluation of others [6], risk-taking [25], negotiation skills [7], and helping behavior [16]. Affective computational priming may be useful in human resource software, project management and decision-making software, and browser and search interfaces.

In addition to affect, computational primes can be used to induce a variety of mental states that influence behavior. While this study focuses on priming affect, we can imagine computationally priming for things such as power, individualism, and collectivism, which have all been shown to influence subsequent behavior.

Another area for future research is examining the length of time in which computational primes influence behavior. Previous findings suggest that experimentally induced affect may last up to 30 minutes [32]. Therefore, we might consider how to effectively schedule the use of computational primes in technology. Future research may not only explore the limits of the duration of computational priming in technology use but also consider ways to computationally prime users the entire time a tool is used.

\section{CONCLUSION}

Affect influences how people feel, think, and act. For this reason, as HCI researchers and designers, it is imperative that we understand the role of affect on the use of technology, which people use to complete their daily work. Although this study focused on the role of affect and creativity tool use in response to a grand challenge posed to the HCI community [36], we see this as a small window into a wealth of opportunities for the human-computer interaction community to understand users and design technology through the lens of affect and behavior. Thirty years ago, by considering the cognitive functioning of users, HCI researchers expanded the understanding of technology from functionality to usability. Now, by integrating an understanding of affective functioning into HCI, we have the opportunity to further advance HCI for increased acceptance, use, and performance. We suggest that understanding how to design technologies such that they mesh with affective functioning is the next critical step for the HCI community. Ultimately, as HCI designers, we will design systems that respond to human affective, cognitive, and behavioral processes.

\section{ACKNOWLEDGEMENTS}

We thank our study participants and expert raters for their time. We especially thank Wendy Ju for helping with participant recruitment. We would also like to thank our reviewers, Joel Brandt, Jeremy Barksdale, and Kyla McMullen for their insightful comments on previous versions of this paper. We appreciate the input received from Adobe's Creative Technologies Lab, Scott Klemmer, and Don Norman on this work. The first author is supported by the National Science Foundation Graduate Research Fellowship.

\section{REFERENCES}

1. Amabile, T. Creativity in Context. Westview Press, Boulder, CO, (1996).

2. Amabile, T. The social psychology of creativity: A componential conceptualization. Journal of Personality and Social Psychology, 45 (1983), 357-376.

3. Amabile, T. Social psychology of creativity: A consensual assessment technique. Journal of Personality and Social Psychology, 43, 5 (1982), 9971013.

4. Amabile, T., Barsade, S., Mueller, J. and Staw, B. Affect and Creativity at Work. Administrative Science Quarterly, 50 (2005), 367-403.

5. Bao, P., Gerber, E., Gergle, D. and Hoffman, D., Momentum: getting and staying on topic during a brainstorm. In Proc. CHI 2010, ACM Press (2010), 1233-1236.

6. Cardy, R.L. and Dobbins, G.H. Affect and appraisal accuracy: Liking as an integratl dimension in evaluating performance. Journal of Applied Psychology, 71 (1986), 672-678.

7. Carnevale, P. and Isen, A. The influence of positive affect and visual access on the discovery of integrative solutions in bilateral negoation. Organizational Behavior and Human Decision Processes, 37 (1986), 1-13.

8. Carver, C. and Scheier, M. Origins and functions of positive and negative affect: a control-process view. Psychological Review, 97 (1990), 19-35.

9. Clore, G. Why emotions are felt. in Ekman, P. and Davidson, R. eds. The nature of emotion: fundamental questions, Oxford University Press, New York, (1994), 103-111.

10. Compeau, D. and Higgins, C.A. Computer SelfEfficacy: Development of a Measure and Initial Test. MIS Quarterly, 19, 2 (1995), 189-211.

11. Czerwinski, M., Horvitz, E. and Wilhite, S., A diary study of task switching and interruptions. In Proc. CHI 2004, ACM (2004), 175-182.

12. Duncker, K. On problem solving. Psychological Monographs, 58, 5 (1945), Whole No. 270.

13. Erez, A. and Isen, A.M. The influence of positive affect on the components of expectancy motivation. Journal of Applied Psychology, 87, 6 (2002), 10551067.

14. Estrada, C., Isen, A. and Young, M. Positive affect improves creative problem solving and influences reported source of practice satisfaciton in physicians. Motivation and Emotion, 18, 4 (1994), 285-299. 
15. Frederickson, B. What good are postive emotions? Review of General Psychology, 2 (1998), 300-319.

16. George, J. and Brief, A. Feeling good-doing good: A conceptual analysis of the mood at work organizational spontaneity. Psychological Bulletin, 112 (1992), 310-329.

17. George, J. and Zhou, J. Understanding when bad mood fosters creativity and good ones don't: The role of context and clarity of feelings. Journal of Applied Psychology, 87 (2002), 687-697.

18. Guilford, J.P. The Nature of Human Intelligence. McGraw-Hill, New York, (1967).

19. Heer, J. and Bostock, M., Crowdsourcing graphical perception: using mechanical turk to assess visualization design. In Proc. CHI 2010, ACM (2010), 203-212.

20. Hewett, T., Czerwinski, M., Terry, M., Nunamaker, J., Candy, L., Kules, B. and Sylvan, E., Creativity Support Tool Evaluation Methods and Metrics. In Creativity Support Tools: A workshop sponsored by the National Science Foundation (2005), 10-24.

21. Ipeirotis, P.G., Provost, F. and Wang, J., Quality management on Amazon Mechanical Turk. In Proc. ACM SIGKDD Workshop on Human Computation, ACM (2010), 64-67.

22. Isen, A. Positive Affect. in Dalgleish, T. and Power, M. eds. The Handbook of Cognition and Emotion, Wiley, New York, (1999), 521-539.

23. Isen, A., Daubman, K. and Nowicki, G.P. Positive affect facilitates creative problem solving. Journal of Personality and Social Psychology, 52 (1987), 11221131.

24. Isen, A., Johnson, M., Mertz, E. and Robinson, G. The influence of positive affect on the unusualness of word associations. Journal of Personality and Social Psychology, 48 (1985), 1413-1426.

25. Isen, A., Nygren, T. and Ashby, G. The influence of positive affect on subjective utility of gains and losses: It's not worth the risk. Journal of Personality and Social Psychology, 55, 710-717 (1988).

26. Izard, C.E. Human Emotions. Plenum Press, New York, (1977).

27. Kauffman, G. and Vosburg, S. The Effects of Mood on Early and Late Idea Production. Creativity Research Journal, 14, 3 (2002), 317-330.

28. Kittur, A., Chi, E.H. and Suh, B., Crowdsourcing user studies with Mechanical Turk. In Proc. CHI 2008, ACM (2008), 453-456.
29. Lang, P., Bradley, M., Cuthbert, B. International Affective Picture System: Affective ratings of pictures and instruction manual, NIMH Center for the Study of Emotion \& Attention, University of Florida, 2008.

30. Lee, K., C. K. and Cassidy, T. Principles of design leadership for industrial design teams in Taiwan. Design Studies, 10, 16 (2007), 1-26.

31. Mednick, M.T., Mednick, S.A. and Mednick, E.V. Incubation of creative performance and specific associative priming. Journal of Abnormal and Social Psychology, 69, 84-88 (1964).

32. Morris, W. Mood: The frame of mind. Springer-Verlag, New York, 1989.

33. Picard, R. Affective computing. The MIT Press, 2000.

34. Puccio, G. and Murdock, M. Creativity Assessment: Readings and Resources. Creative Education Foundation, Buffalo, NY, (1999).

35. Ross, J., Irani, L., Silberman, M.S., Zaldivar, A. and Tomlinson, B., Who are the crowdworkers?: shifting demographics in mechanical turk. In Proc. Ext. Abstracts CHI 2010, ACM (2010), 2863-2872.

36. Shneiderman, B. (ed.), Creativity Support Tools: A Grand Challenge for HCI Researchers, London, 2009.

37. Shneiderman, B., Fischer, G., Czerwinski, M., Resnick, M., Myers, B., Candy, L., Edmonds, E., Eisenberg, M., Giaccardi, E., Hewett, T., Jennings, P., Kules, B., Nakakoji, K., Nunamaker, J., Pausch, R., Selker, T., Sylvan, E. and Terry, M. Creativity Support Tools: Report From a U.S. National Science Foundation Sponsored Workshop. International Journal of Human-Computer Interaction, 20, 2 (2006), 61-77.

38. Simonton, D.K. Origins of Genius: Darwinian Perspective on Creativity. Oxford University Press, New York, (1999).

39. Slepian, M., Weisbuch, M., Rutchick, A., Newman, L. and Ambady, N. Shedding light on insight: Priming bright Ideas. Journal of Experimental Social Psychology, 46 (2010), 696-700.

40. Tierney, P. and Farmer, S. Creative self-efficacy: potential antecedents: Potential antecedents and relationship to creative performance. Academy of Management Journal, 45 (2002), 1137-1148.

41. Torrance, E.P. Torrance Tests of Creative Thinking. Scholastic Testing Services, (1974).

42. Zajonc, R.B. Feeling and thinking: Preferences need no inferences. American Psychologist, 35, 2 (1980), 151175. 